\title{
Loss-based process capability indices: a review
}

\begin{abstract}
Process capability indices (PCIs) are appropriate tools to measure the inherent capability of a process, but most of them do not consider the losses of a process, while in today's competitive business environment, it is becoming more and more important for companies to evaluate and minimise their losses. This paper presents a review of loss-based PCIs such as Cpm, C pmk, PCId, Cpc, Le and L"e. This paper also discusses characteristics of loss-based PCIs such as reject based, asymmetric, bounded, loss based and target based. Finally, recommendations were made for developing a new loss-based process capability index with more excellent specifications.
\end{abstract}

Keyword: Loss-based process capability indices; PCIs; Process capability indices; Specifications 